\title{
A Novel Design: Porous Boundary Condition for Drag Reduction of Three Dimensional Rotating Body of Revolution-Slender Body
}

\author{
K. Alhussan
}

Deputy Director of Space Research Institute, King Abdulaziz City for Science \& Technology, Riyadh, Saudi Arabia

Email: alhussan@kacst.edu.sa

\begin{abstract}
The work to be presented herein is a Computational Fluid Dynamics investigation of the complex fluid mechanisms that occur over slender body by using a porous boundary condition as a decelerator, specifically with regard to the total aerodynamic drag. Drag is needed to decelerate the body. The aim of this research is to design deceleration devices for high-speed rotating slender body. In this paper a qualitative analysis of the flow structure over a slender body with porous boundary condition as decelerator was shown. This paper discusses research variables that directly impact the ability to obtain turbulent flow and techniques to increase flow separation.

The Results of contour plots of Mach number for different angles of attack, see Figures 1 and 2, namely from $0^{\circ}$ to $20^{\circ}$, for free-stream Mach number of 3.0 and for angular velocity of $10000 \mathrm{RPM}$, will demonstrate that the aerodynamic forces and the stream paths of the velocity vectors are changed when the porous boundary conditions are integrated with the slender body. One can reveal from this study a novel design for a deceleration device of a high-speed slender body. The geometrical parameters are developed in this paper as shown in figure 1 . This study shows that the novel design of the decelerator will be optimized under these flow conditions; Mach number of 3.00, the Mach number ratio is in the range of 0.6 to 0.8 , and angle of attack between $10^{\circ}$ and $20^{\circ}$.
\end{abstract}

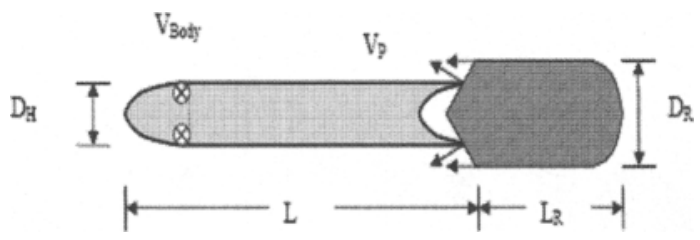

Figure 1: A schematic view of high-speed body of revolution with deceleration device

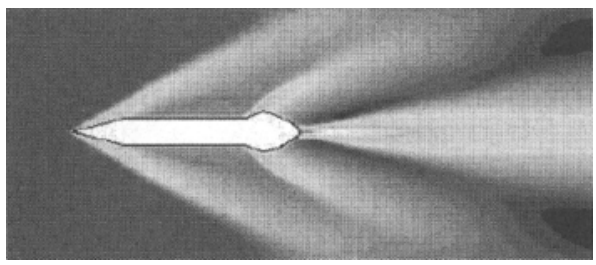

Figure 2: Contour plots of Mach number

\section{REFERENCES}

1. Alhussan K. Supersonic flow over blunt body with a decelerator. Journal of IASME Transaction Issue 3, August 2005; 1: 98-104

2. Alhussan $K$. Oblique shock waves interaction in a non-steady three dimensional rotating flow. In: Proceedings of FEDSM2005 ASME Fluids Engineering Division Summer Meeting and Exhibition FEDSM2005-77442 June 19-23, 2005; Houston, TX, USA

3. Patankar S. Numerical Heat Transfer and Fluid Flow. Series in Computational Methods in Mechanics and Thermal Sciences, Tayler \& Francis, 1980 